\title{
Right Ventricular Failure Following Acute Type A Aortic Dissection: An Alternative Strategy
}

\author{
Ruchir P. Mashar ${ }^{1}$, Rashmi P. Birla ${ }^{2}$, Paul D. Waterworth ${ }^{3 *}$ \\ ${ }^{1}$ General Surgery, Hereford County Hospital, Hereford, UK \\ ${ }^{2}$ Cardiothoracic Surgery, Liverpool Heart and Chest Hospital, Liverpool, UK \\ ${ }^{3}$ Cardiac Surgery, Wythenshawe Hospital, Manchester, UK \\ Email: *ruchirmashar822@hotmail.com
}

How to cite this paper: Mashar, R.P., Birla, R.P. and Waterworth, P.D. (2019) Right Ventricular Failure Following Acute Type A Aortic Dissection: An Alternative Strategy. World Journal of Cardiovascular Surgery, 9, 35-39.

https://doi.org/10.4236/wjcs.2019.93004

Received: July 2, 2018

Accepted: March 26, 2019

Published: March 29, 2019

Copyright $\odot 2019$ by author(s) and Scientific Research Publishing Inc. This work is licensed under the Creative Commons Attribution International License (CC BY 4.0).

http://creativecommons.org/licenses/by/4.0/

\begin{abstract}
Coronary malperfusion is a complication of aortic dissection, and can lead to ventricular failure. We report 2 cases of right ventricular (RV) failure, following aortic dissection, successfully bridged to recovery using right ventricular assist devices (RVADs). The first was a 54 year-old man, who had difficulty weaning from bypass due to a stunned RV following aortic root replacement. The second was a 34 year-old woman, who after replacement of the ascending aorta, suffered episodes of ventricular fibrillation (VF), and RV failure. Both had an RVAD implanted, allowing eventual recovery. We recommend more widespread use of VADs in such cases.
\end{abstract}

\section{Keywords}

Right Ventricular Failure, Assist Devices, Acute Type A Aortic Dissection

\section{Introduction}

Coronary malperfusion is a complication of acute aortic dissection [1], causing difficulty in weaning from bypass. The use of inotropes, interventional strategies such as intra-aortic balloon pumps, and coronary artery bypass grafting is well documented [1]. However, in refractory cases, mechanical circulatory support in the form of ventricular assist devices may be the only option. We present two cases of acute aortic dissection, in which an RVAD was successfully used for the failing right ventricle.

\section{Case Presentations}

\subsection{Case 1}

A 54 year-old man presented with an acute type A aortic dissection. 
Trans-oesophageal echocardiography (TOE) confirmed a tear in the aorta just above the sinotubular junction with no aortic regurgitation.

Intra-operatively, the tissues were found to be very friable. The ascending aorta was replaced with an interposition graft (28 mm Hemashield Platinum). On weaning from cardiopulmonary bypass (CPB), bleeding was noted from the proximal anastomosis. Surgical repair was attempted, but the friable tissues did not lend themselves to a haemostatic seal. CPB was reinstituted and aortic root replacement was performed with a St Jude mechanical aortic valve conduit (23 $\mathrm{mm}$ valve). The patient was weaned from $\mathrm{CPB}$ on moderate inotropic support and primary closure of the chest was performed.

Within 5 minutes of closure, $500 \mathrm{mls}$ of blood had drained into the drains and the blood pressure fell to a systolic of $60 \mathrm{mmHg}$. On emergency reopening, bleeding was noted from the right coronary button, which was torn. CPB was reinstituted for the third time and the right coronary artery (RCA) was grafted using the long saphenous vein (LSV). The right coronary ostium was clipped and oversewn. The total cardiopulmonary bypass, the cross-clamp and the circulatory arrest times were recorded as 321 minutes, 221 minutes and 21 minutes respectively. Myocardial protection was achieved using cold blood cardioplegia delivered intermittently throughout.

TOE now showed good left ventricular (LV) function but a stunned right ventricle (RV). An intra-aortic balloon pump (IABP) was inserted and subsequent wean from CPB was successful on inotropic support. RV function remained poor however, and the patient developed VF, requiring defibrillation. To support the right ventricle, a RVAD (Levitronix Centri Mag) was implanted. This allowed stabilisation of his haemodynamic parameters and facilitated weaning from bypass. TOE on day 21 showed good overall RV function, with septum, apex and infundibulum moving well, allowing a successful wean from the RVAD the same day. The patient was discharged from ITU on day 23 and discharged home on day 25 . He remains well and at clinic follow up at 5 months had returned to his hobby of regular cycling. An outpatient echocardiogram showed normal LV function, a well-functioning prosthetic aortic valve and normal RV size with preserved radial contraction.

\subsection{Case 2}

A 34 year-old woman was referred from a district general hospital following coronary artery angioplasty. At the time of the angioplasty, the cardiologist thought that he had created an RCA dissection, and therefore he stented the RCA. The dissection was thought to extend into the aortic root, and hence she was referred on for further management. On further questioning, the patient had a 3-day history of chest and back pain. A bedside transthoracic echocardiogram (TTE) raised suspicion of an aortic dissection, subsequently confirmed by computed tomography (CT) (see Figure 1).

The patient was taken to theatre where $\mathrm{CPB}$ was instituted via left femoral cannulation, and the patient was systemically cooled to $15^{\circ} \mathrm{C}$. Intraoperatively, 


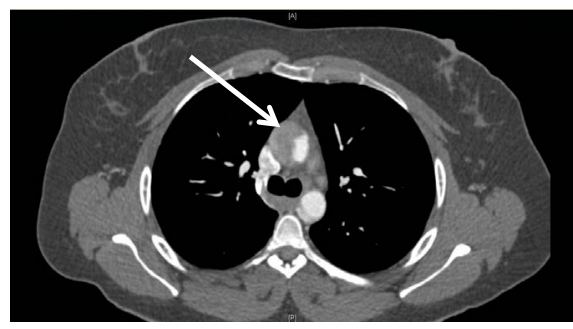

(a)

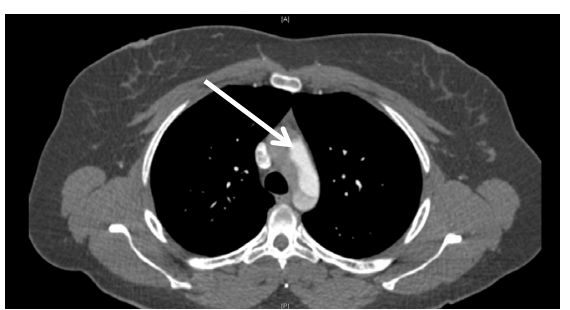

(b)

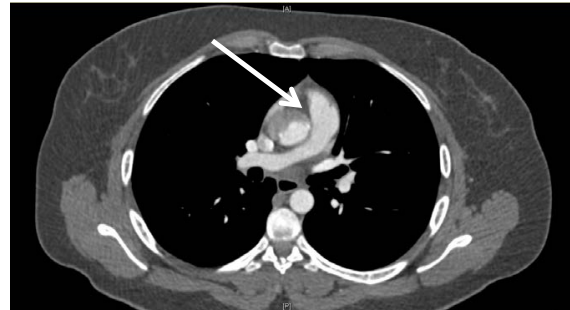

(c)

Figure 1. Computed tomography (CT) images demonstrating the double lumen (a) with intra-mural contrast ((b) and (c)), confirming a suspected aortic dissection.

discolouration of the ascending aorta was noted suggesting a type A aortic dissection. No intimal tears were identified in the aortic arch, with a morphologically normal aortic valve. However a dissection was noted in the right coronary ostium. An interposition graft (16 mm Hemashield Platinum) was constructed with an open distal anastomosis. The RCA was grafted using the LSV, with the ostium oversewn and pledgeted. The bypass, cross-clamp, and the circulatory arrest times were 172 minutes, 128 minutes, and 20 minutes respectively. Myocardial protection was achieved using cold blood cardioplegia delivered intermittently.

On day 1, continued episodes of VF and RV failure were noted requiring high inotropic support and hence she underwent insertion of a percutaneous RVAD (Levitronix CentriMag), causing immediate haemodynamic improvement with reduction of inotropic requirements. On day 6, RVAD flows were noted to decrease despite multiple fluid boluses. A bedside TTE showed a global pericardial collection, with an akinetic RV free wall. The chest was reopened as an emergency on ITU, and large clots evacuated from the anterior surface of the RV. On day 11, once again persistent hypotension was noted, with TTE confirming a 4 $\mathrm{cm}$ loculated effusion, and empty biventricular cavities. The patient underwent re-exploration in theatre with drainage of a pericardial effusion, clots and bilateral pleural effusions. Following this, she was successfully extubated on day 12 , and made a gradual recovery, with a bedside TTE on day 20 showing impaired longitudinal but good RV contractions. The RVAD was explanted on day 22. She was transferred to the ward on day 24 , and discharged home on day 28 . She remains well however when reviewed in clinic 7 months later, was dependent on sildenafil and had some reduced exercise tolerance. 


\section{Discussion}

RV failure following aortic dissection repair can be difficult to manage and may not respond to methods such as the use of inotropes, RCA bypass surgery and intra-aortic balloon pumps. We describe two cases demonstrating the lifesaving use of RVADs in refractory circumstances. In our first case, an RVAD was successfully used to rescue a stunned RV. Our second case was noted to have recurrent VF and RV failure, which was once again, successfully treated with a percutaneous RVAD.

We have found similar reports of RVAD of effective utilisation of RVAD implantation in cases of RV failure following cardiotomy [2]. Their temporary use has been suggested in RV failure following cardiac surgery to bridge recovery [3]. There have however been limited reports of their use following acute type A aortic dissection. Asakura et al. report their experience in which 3 out of 9 patients survived using left sided mechanical support following repair of acute type A aortic dissection [1]. Kolesar et al. similarly report the use of a left ventricular assist device in a pregnant woman with acute aortic dissection [4]. There is only one report of the successful utilisation of an RVAD in RV failure following an aortic dissection repair. This reports its use in a 64 year-old woman, to facilitate weaning from bypass following a repair with a $30 \mathrm{~mm}$ gel-sealed Dacron graft, who likewise made a full recovery [5].

A major hazard to RVAD use remains the concomitant need for anticoagulation, especially after dissection repair. Of the two cases described above, one required multiple urgent re-explorations, evacuation of pericardial clots and collections. Nevertheless, these cases demonstrate that RVADs can be used successfully to manage RV failure following type A aortic dissection and should be considered when available.

As technology advances, and centres develop more experience of using temporary mechanical assist devices, it may become common for more cardiac surgery centres to offer LVAD/RVAD support following cardiac surgery.

\section{Consent}

Informed consent was obtained from both patients whose cases are described in this report.

\section{Conflicts of Interest}

The authors declare no conflicts of interest regarding the publication of this paper.

\section{References}

[1] Asakura, T., et al. (2007) Coronary Malperfusion Due to Acute Type A Aortic Dissection; Surgical Strategy and Results. Kyobu Geka, 60, 297-302.

[2] Moazami, N., et al. (2004) Mechanical Support for Isolated Right Ventricular Failure in Patients after Cardiotomy. The Journal of Heart and Lung Transplantation, 
23, 1371-1375. https://doi.org/10.1016/j.healun.2003.09.022

[3] Lang, S.A., O’Neill, B., Waterworth, P. and Bilal, H. (2014) Can the Temporary Use of Right Ventricular Assist Devices Bridge Patients with Acute Right Ventricular Failure after Cardiac Surgery to Receovery? Interactive CardioVascular and Thoracic Surgery, 18, 499-510. https://doi.org/10.1093/icvts/ivt472

[4] Kolesar, A., Sabol, F., Luczy, J. and Bajmoczi, M. (2010) Use of Left Ventricular Assist Device in a Pregnant Woman with Acute Aortic and Coronary Dissections. Interact. Interactive Cardio Vascular and Thoracic Surgery, 11, 194-195. https://doi.org/10.1510/icvts.2010.238501

[5] Luciani, G.B., Fabbri, A., Faggian, G., Marino, P. and Mazzucco, A. (1993) Mechanical assist device for right ventricular failure after aortic dissection. The Annals of Thoracic Surgery, 56, 363-366. https://doi.org/10.1016/0003-4975(93)91178-P 